\title{
Enhanced Kat3A/Catenin transcription: a common mechanism of therapeutic resistance
}

\author{
Andrea Bild ${ }^{1}$, Jia-Ling Teo ${ }^{2}$, Michael Kahn² \\ 'Department of Medical Oncology \& Therapeutics Research, Beckman Research Institute of the City of Hope, Duarte, CA 91010, \\ USA. \\ ${ }^{2}$ Department of Molecular Medicine, Beckman Research Institute of the City of Hope, Duarte, CA 91010, USA.
}

Correspondence to: Prof. Michael Kahn, Department of Molecular Medicine, Beckman Research Institute of the City of Hope, 1500 East Duarte Road, Flower Building, Duarte, CA 91010, USA.E-mail: mkahn@coh.org

\begin{abstract}
How to cite this article: Bild A, Teo JL, Kahn M. Enhanced Kat3A/Catenin transcription: a common mechanism of therapeutic resistance. Cancer Drug Resist2019;2:917-32. http://dx.doi.org/10.20517/cdr.2019.32
\end{abstract}

Received: 22 Apr 2019 First Decision: 13 May 2019 Revised: 4 Jun 2019 Accepted: 18 Jun 2019 Published: 19 Sep 2019

Science Editor: Helen M. Coley Copy Editor: Han-juan Zhang Production Editor: Jing Yu

\begin{abstract}
Cancers are heterogeneous at the cellular level. Cancer stem cells/tumor initiating cells (CSC/TIC) both initiate tumorigenesis and are responsible for therapeutic resistance and disease relapse. Elimination of CSC/TIC should therefore be able to reverse therapy resistance. In principle, this could be accomplished by either targeting cancer stem cell surface markers or "stemness" pathways. Although the successful therapeutic elimination of "cancer stemness" is a critical goal, it is complex in that it should be achieved without depletion of or increases in somatic mutations in normal tissue stem cell populations. In this perspective, we will discuss the prospects for this goal via pharmacologically targeting differential Kat3 coactivator/Catenin usage, a fundamental transcriptional control mechanism in stem cell biology.
\end{abstract}

Keywords: Kat3 coactivator, CREB-binding protein, p300, therapy resistance, stem cell, cancer stem cell

\section{INTRODUCTION}

Cancer is a major contributor to worldwide mortality ${ }^{[1]}$. There are minimally four broad resistance-inducing strategies that are employed by cancer cells including: (1) direct target reactivation; (2) activation of signals upstream or downstream of oncogenes; (3) engagement of parallel oncogenic pathways; and (4) adaptive survival mechanisms. Despite tremendous advances in targeted therapeutics and personalized medicine, which have significantly increased progression free survival, maximum clinical success as defined by 
overall survival or "cures", remain limited due to therapeutic resistance ${ }^{[2]}$. These resistance mechanisms can be attributed to a subpopulation of self-renewing, highly tumorigenic, drug-resistant cancer stem cell/ tumor initiating cell (CSC/TIC), in which therapeutic pressure leads to the selection of therapy resistant clone $^{[3-8]}$.

\section{Stem cells and cancer stem cells}

All stem cells by definition, have the capacity to both self-renew (i.e., make at least one identical copy of itself at each division) as well as to differentiate into more mature, albeit less potent, specialized cells. The concept of CSC is not new. Cohnheim, more than 150 years ago, proposed that cancer might arise from rare cells with stem cell-like properties ${ }^{[9]}$. The existence of CSC has now been demonstrated in many tumor types including leukemia, brain, breast, bladder, prostate, colon, etc., where their presence has been associated with disease recurrence, multidrug resistance and metastasis ${ }^{[10]}$. Therefore a critical goal to change the course of cancer therapy is to develop strategies to safely eliminate CSC without deleterious effects to normal spermatogonial stem cell (SSC) populations.

Two mechanisms are proposed to account for the generation of CSC. In the stochastic model, cancer cell plasticity endows non-CSC with the ability to dedifferentiate into CSC. Alternatively, in the hierarchical model, CSC are able to self-renew thereby expanding the CSC pool from which escape mutants can be selected. CSC and SSC share multiple characteristics, including self-renewal and the potential to differentiate. As previously pointed out, the term "cancer stem cell" does not have to refer to the cell of origin $^{[11]}$. Rather the term CSC refers to cells that have "stem-like" properties. CSC can originate from tissue stem cells, transiently amplifying cells or potentially even differentiated cells ${ }^{[12]}$. SSC, due to their longevity and self-renewing properties, have a far greater propensity to accumulate carcinogenic mutations, which could markedly influence the behavior of those cells, e.g., accelerate self-renewal via a switch from asymmetric to symmetric division, which will be further discussed ${ }^{[13,14]}$. It is also possible that the initial mutations occur in SSC, yet the final mutations that confer oncogenesis occur during neoplastic transformation in downstream progeny that have blocks in terminal differentiation ${ }^{[15]}$. Further, interaction with the environment or signaling changes within a cell can lead to epigenetic or phenotypic state changes relevant to CSC generation ${ }^{[16]}$. Regardless of the exact origin of CSC, therapeutic resistance in CSC has been associated with (1) quiescence, as most conventional cytotoxic agents target proliferating cell ${ }^{[17,18]}$; (2) high expression of drug-efflux pumps, e.g., ATP binding cassette (ABC) family transporters ${ }^{[19]}$; (3) increased DNA repair and detoxifying enzymes ${ }^{[20]}$; (4) acquisition of an EMT-like phenotype ${ }^{[21]}$; and (5) utilization of hypoxic niche microenvironments that provide survival fostering signals ${ }^{[22]}$.

Targeting CSC could in principle be accomplished via the targeting of CSC specific cell surface markers or through alternatively "stemness" pathways. Although the successful therapeutic elimination of "cancer stemness" offers enormous promise, it will require significant precision to avoid deleterious effects (e.g., depletion of, or increases in, somatic mutations) in normal SSC populations. Unfortunately in this regard, the similarities between normal adult SSC and CSC far outweigh their differences ${ }^{[23]}$. CSC express similar "stemness" markers and exhibit similar cellular behaviors to SSC as described above. SSC in tissues preferentially inhabit specialized hypoxic niches and are critical for both normal tissue homeostasis and regeneration after injury ${ }^{[24-26]}$. Long-lived SSC are quiescent and rarely become activated under homeostatic conditions, however upon injury to repair damaged tissue, they enter the cell cycle. CSC occupy the same hypoxic niches, thereby competing with normal SSC for this limited environment. The same signal transduction pathways utilized in SSC maintenance, proliferation and differentiation (i.e., Wnt, Notch, Hedeghog, TGFß/BMP, JAK/Stat, Hippo, FGF/MAPK/PI3K) also regulate CSC ${ }^{[27-29]}$. For both CSC and SSC, there are multiple points of intersection and crosstalk, including feedback and feed forward loops, connecting the various signaling cascades that modulate "stemness" allowing for escape from driver directed therapeutics. These targets and therapies blocking these pathways are summarized in recent reviews ${ }^{[8,30-32]}$. 


\section{Asymmetric Division}
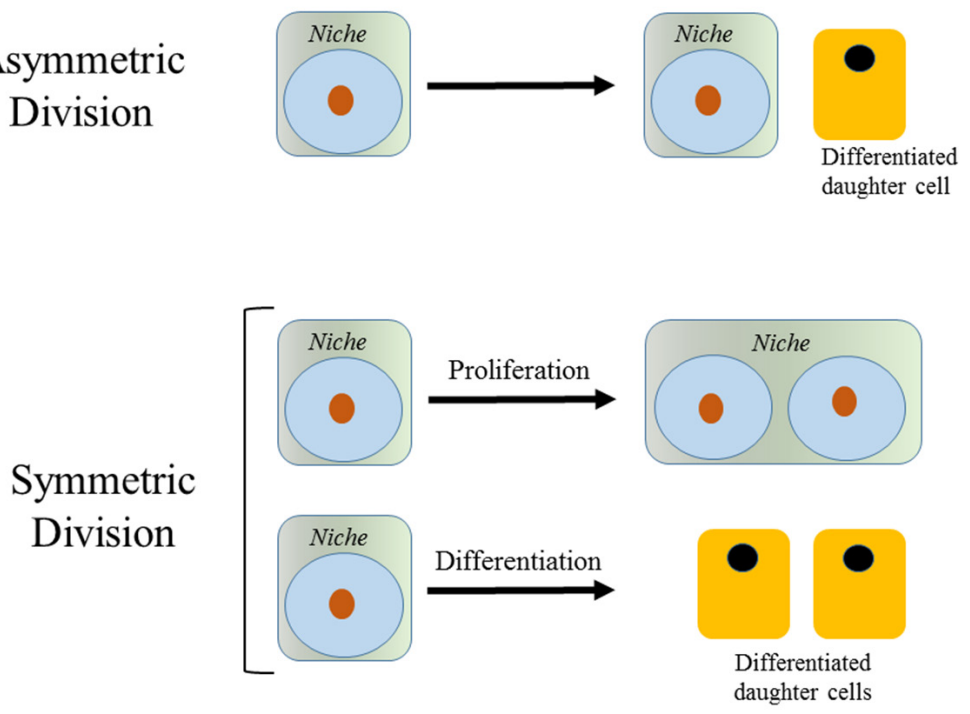

Figure 1. Stem cell divisions. An asymmetric division results in the production of two daughter cells with different cell fates-one a stem cell and the other a diafferentiated daughter cell. There are two modes of symmetric divisions: symmetric non-differentiative divisions generate two daughter cells that remain as stem cells, whereas symmetric differentiative division gives rise to two daughter cells, both of which are differentiated daughter cells

Adult SSC are present in limited numbers. They are believed to be essentially immortal and remain with us for our entire lives. The "dark side" of the immortality of SSC is their capacity to be corrupted into CSC. Like their normal counterpart SSC, CSC exhibit self-renewal capacity and differentiation potential, albeit with aberrant and incomplete differentiation, thereby having the capacity to maintain or renew and propagate a tumor. Under normal homeostatic conditions, long-term SSC divide relatively infrequently, perhaps only once every few months ${ }^{[33]}$ or even less ${ }^{[34]}$. Quiescent SSC, once they enter the cell cycle, can undergo mitosis to give rise to two daughter cells. Mitotic stem cells can divide either symmetrically or asymmetrically [Figure 1]. Ideally, an asymmetric balance is maintained, whereby one of the daughter cells remains in its niche as a stem cell and the other daughter proceeds forward to amplify and subsequently differentiate. However, stem cells (both SSC and CSC) can also undergo symmetric divisions. There are two modes of symmetric division: (1) symmetric non-differentiative divisions, where both daughter cells remain as stem cells in their niche; or (2) symmetric differentiative divisions, where both cells go on to differentiate [Figure 1]. Symmetric division in our essentially "immortal" SSCs, are considered deleterious, leading either to premature exhaustion of the stem cell pool or alternatively increasing the number of DNA lesions accumulated in SSC (via symmetric differentiative and non-differentiative divisions respectively). The preference for long-lived SSC to undergo asymmetric divisions is outlined in the Cairn's "immortal strand hypothesis" ${ }^{3[35]}$, which postulated that the stem cell desires to retain its original uncopied strands of DNA and to pass on the duplicated strands that contain multiple copy errors, inherent in the DNA replication process, to its differentiated daughter cell, thereby minimizing the total number of DNA mutations that accumulate in the long-lived SSC population. In order to make the decision to divide symmetrically versus asymmetrically, a stem cell undergoing mitosis must read an enormous array of information from its environment (e.g., oxygen levels, nutrient levels, circadian cycles, growth factors, adhesion molecules, kinase cascades, cell-cell contacts, etc.). How is all of this information integrated to decide a stem cell's fate, i.e., to exit quiescence and subsequently divide either asymmetrically or symmetrically, be it a normal SSC or a CSC?

Interestingly, a preference for symmetric over asymmetric divisions appears to be one of the fundamental differences between CSC and SSC. Breast cancer stem cells with p53 mutations preferentially undergo 
symmetric divisions ${ }^{[36]}$. Loss of the tumor suppressor PTEN leads to premature exhaustion of the normal hematopoietic stem cell population, presumably via increased symmetric differentiative divisions and expansion of the leukemic stem cell population via increased non-differentiative symmetric divisions ${ }^{[37]}$. Indirect perturbation of Notch signaling, via genetic activation of the Hedgehog pathway, also causes an increase in neural stem cell symmetric divisions ${ }^{[38]}$. Symmetric differentiative divisions by "corrupted" SSC prior to the accumulation of additional deleterious mutations generates bona fide CSCand can stochastically eliminate this SSC population. This mechanism prevents non-differentiative symmetric divisions expanding the "pre-CSC" pool. An example of this expansion of the "pre-CSC" pool is represented by clonal hematopoiesis of indeterminate potential (CHIP). CHIP is defined by the presence of somatic hematologic-cancer-associated gene mutations and can be seen in the peripheral blood of at least $10 \%$ of people older than 60 years of age without any history of hematologic disorders ${ }^{[39]}$. The presence of CHIP is associated with an increased risk of hematologic cancers and an increased overall mortality ${ }^{[40]}$.

\section{Wnt/Catenin-dependent transcription and "stemness"}

Wnt signaling is an ancient and highly evolutionarily conserved pathway that is important throughout embryonic development and the life of an organism. It is a very complex signaling cascade ${ }^{[4]}$ that initiates a broad range of intracellular responses broadly classified as either canonical (involving nuclear $\beta$-catenin mediated transcription) or non-canonical (planar cell polarity, $\mathrm{Ca}^{2+} / \mathrm{PKC}$ activation) ${ }^{[42,43]}$. Canonical Wnt signaling is generally associated with proliferation and lack of differentiation (for example in cancer), whereas the non-canonical pathway regulates cellular patterning and tissue organization. $\beta$-catenin is critical in both pathways via its roles either in the nucleus or cytoskeleton and cytoplasmic membrane, respectively. Although designating Wnt signaling as either canonical or non-canonical allows for simplified conceptual discourse, there is great crosstalk between the two responses, and Wnt crosstalk regulates complex nonlinear networks in development and homeostasis ${ }^{[4]}$. Nuclear $\beta$-catenin, although additional catenins, including $\gamma$-catenin/plakoglobin, may additionally participate under particular circumstances ${ }^{[45]}$, in transcription is controlled by the so-termed "canonical Wnt" or "Wnt/ $\beta$-catenin" signaling cascade. Nuclear translocation of $\beta$-catenin and its subsequent transcriptional activity can also be induced by nonWnt signaling. Epithelial to mesenchymal transition, leads to $\beta$-catenin nuclear translocation ${ }^{[46]}$, perhaps through down-regulation of $\beta$-catenin's cytoplasmic binding partner E-cadherin ${ }^{[47]}$. Receptor tyrosine kinases $^{[48]}$ and non-receptor tyrosine kinases including $\operatorname{Src}^{[49]}$ and $\mathrm{Abl}^{[50]}$ can enhance $\beta$-catenin-mediated transcription by disrupting the E-cadherin/ $\beta$-catenin interaction. Prostaglandins ${ }^{[51]}$, hypoxia ${ }^{[52,53]}$, high glucose levels ${ }^{[54]}$, and cholinergic innervation ${ }^{[55]}$ additionally may activate Wnt/ $\beta$-catenin signaling. A wide range of inputs an influence $\beta$-catenin dynamics and $\beta$-catenin-dependent transcription ${ }^{[56-58]}$. Balancing self-renewal versus differentiation in SSC, requires signaling from a number of other pathways (e.g., Notch, Hedgehog, JAK/Stat, BMP, Hippo, FGF/MAPK) that must be integrated with nuclear $\beta$-catenin signaling [Figure 2]. Wnt signaling is critical in stem cell biology and development ${ }^{[59]}$. However, there is no consensus on whether Wnt signaling is important for either maintenance of potency ${ }^{[3,60]}$ or the differentiation of stem cells $^{[6]]}$. Wnt/catenin signaling clearly plays dichotomous roles in SSC biology ${ }^{[62]}$.

\section{Wnt/Catenin signaling in cancer stem cells and cancer}

Wnt signaling plays a critical role in SSC homeostasis ${ }^{[63]}$. Not surprisingly, aberrant regulation of Wnt signaling is a recurrent theme in cancer biology ${ }^{[64,65]}$ and has been implicated in the tumorigenic potential of stem cells.

Continued expression of BIRC5/Survivin, a Wnt target gene, in hES cells is essential for teratoma formation ${ }^{[66]}$. Wnt/ $\beta$-catenin regulation of telomerase activity endows stem cells and cancer stem cells with unlimited self-renewal capacity ${ }^{[67]}$. Slug, a strong inducer of EMT in tumors, is associated with nuclear accumulation of transcriptionally active $\beta$-catenin ${ }^{[68]}$. Over-expression of either of the putative Wnt target gene EMT inducing factors twist and snail increases the expression of CSC markers ${ }^{[69]}$. The connection 


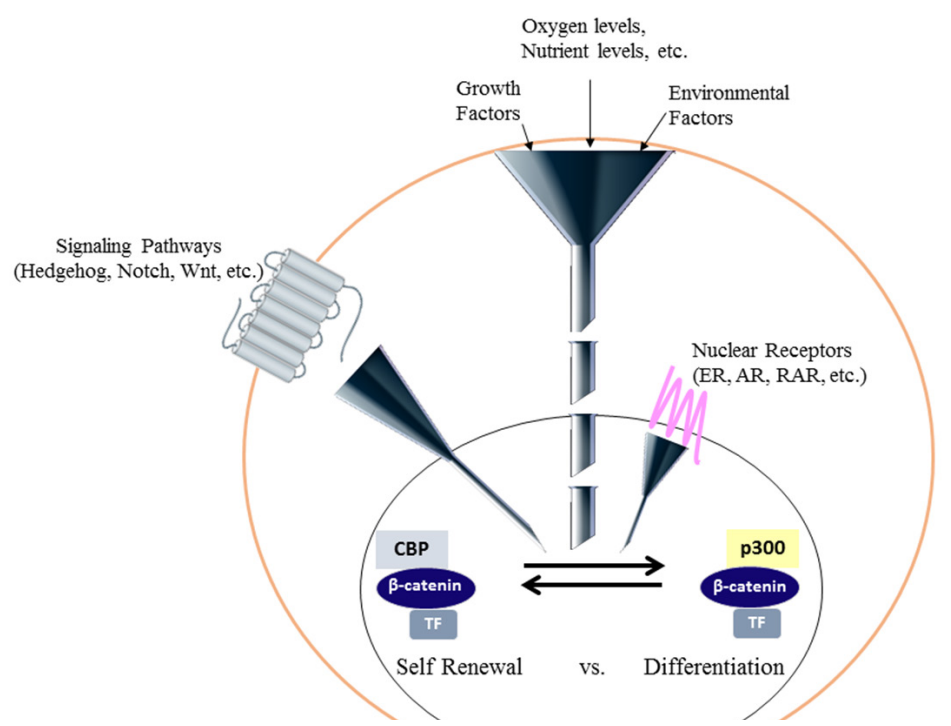

Figure 2. Coordination and integration of multiple signaling cascades is required to regulate the decision of a stem cell to either differentiate or self-renew. Multiple signaling inputs both intrinsic and extrinsic, including nutrient and oxygen levels, growth factors and various signaling cascades must be integrated and funneled down to regulate a transcriptional program either leading to self-renewal or the initiation of differentiation

between enhanced nuclear $\beta$-catenin signaling and EMT is strengthened further by the significant number of $\beta$-catenin target genes (e.g., S100A4, fibronectin, L1CAM, CD44, MMP7, uPAR, etc.) associated with invasion, migration and metastases ${ }^{[70]}$. Wnt signaling in CSC is associated with metastasis ${ }^{[7]]}$, and the regulation of organ specific tropism of CSC during metastasis ${ }^{[72]}$, as well as in the formation of the pre-metastatic niche that nurtures metastasizing $\operatorname{CSC}^{[73]} \cdot \mathrm{Cdx}^{\left[{ }^{[74]}\right.}$ and $\operatorname{Id} 2^{[75]}$, two transcription factors associated with the maintenance of a stem like" state, have been shown to be $\beta$-catenin regulated. Many cell surface markers in stem cell and cancer stem cells are direct Wnt targets, including LGR5/GPR49 ${ }^{[76]}$, $\mathrm{CD} 44^{[77]}, \mathrm{CD} 24^{[78]}, \mathrm{CD} 133^{[79]}, \mathrm{ABC}$ cassette genes ${ }^{[80,81]}$ and $\mathrm{EpCAM}^{[82]}$. The first identified CSC in solid tumors had a $\mathrm{CD} 44^{\text {high }} \mathrm{CD} 24^{\text {low }}$ phenotype and comprised a population of breast cancer CSC possessing tumor-initiating capacity ${ }^{[83]}$. These genes and related references are listed in Table 1. Many Wnt signaling related genes are up-regulated in hematopoietic malignancies ${ }^{[84,85]}$ and epigenetic silencing of negative regulators of the Wnt signaling cascade is frequently associated with leukemia ${ }^{[86]}$. Moreover, aberrant activation of tumor associated Wnt/ $\beta$-catenin signaling has been correlated with resistance to radiation, cytotoxic and targeted chemotherapy ${ }^{[87,88]}$ and most recently checkpoint immunotherapy resistance in multiple tumor types including, melanoma, bladder and head and neck cancers ${ }^{[89]}$. Tumor-intrinsic Wnt/ $\beta$-catenin signaling mediates cancer immune evasion by preventing T-cell and/or dendritic cell infiltration, migration and function, and thereby resistance to immune checkpoint inhibitors ${ }^{[90,91]}$ and has been shown to maintain T-cells in a differentiated exhausted dysfunctional state ${ }^{[92]}$.

\section{Targeting Wnt/Catenin signaling in SSC and CSC}

Successful pharmacologic manipulation of aberrant catenin-regulated transcription of endogenous "stemness" in SSC and CSC holds enormous potential. However, significant concerns arise in regards to potential deleterious effects on normal SSC populations, including increasing DNA lesions or elimination of normal SSC while attempting to eliminate CSC or activate quiescent or senescent SSC ${ }^{[23,41,93]}$. It may seem obvious to target the Wnt signaling pathway in both SSC and CSC and indeed this has engendered substantial efforts to develop therapeutic agents. Despite these efforts, no therapeutic agents to date specifically targeting the Wnt pathway have been approved for use in patients. A number of factors have thwarted progress in this regard. First, the Wnt signaling cascade is highly complex ${ }^{[41,42]}$. For example, in addition to classical canonical Wnt/ $\beta$-catenin/TCF transcription, Wnt proteins elicit a variety of alternative 
Table 1: Wnt Target Genes Associated with "Stemness"

\begin{tabular}{lc}
\hline Genes & References \\
\hline Stemness related & \\
Survivin/BIRC5 & {$[58]$} \\
htert & {$[59]$} \\
Cdx1 & {$[66]$} \\
Id2 & {$[67]$} \\
LGR5/GPR49 & {$[68]$} \\
CD44 & {$[69]$} \\
CD24 & {$[70]$} \\
CD133 & {$[71]$} \\
ABC cassette genes & {$[72,73]$} \\
EpCAM & {$[74]$} \\
EMT related & \\
Slug & {$[60]$} \\
S100A4 & {$[62]$} \\
Fibronectin & {$[62]$} \\
L1CAM & {$[62]$} \\
CD44 & {$[62]$} \\
MMP7 & {$[62]$} \\
uPAR & {$[62]$} \\
\hline
\end{tabular}

non-canonical responses ${ }^{[94,95]}$. Secondly, crosstalk from various non-Wnt factors can also modulate nuclear $\beta$-catenin accumulation as previously discussed. Overall, the ability to target Wnt signaling holds enormous potential; however, like the sword of Damocles, it brings substantial risks and concerns as it is also a crucial pathway in normal SSC maintenance and tissue homeostasis.

\section{Differentiation therapy}

All-trans retinoic acid provided a breakthrough differentiation therapy for acute promyelocytic leukemia. However, broad scale success with differentiation therapy has not been achieved to date ${ }^{[96]}$. As stated previously, a preference for symmetric over asymmetric divisions appears to be one of the fundamental differences between CSC and SSC. The question then is: can we safely manipulate endogenous stem cell populations by taking advantage of their preferred modes of division to differentiate away the CSC population without eliminating normal SSCs?

In order to form a transcriptionally active complex, $\beta$-catenin must recruit one of the two Kat3 transcriptional coactivators, Kat3A, cAMP response element binding protein [CREB-binding protein (CBP)] or its closely related homolog Kat3B, p300 (E1A-binding protein, $300 \mathrm{KDa}$ ) ${ }^{[43,97]}$ to promoters and enhancers. Kat3 coactivators, by binding to hundreds of proteins, play critical roles as master regulators of transcription. Kat3 activation has been previously reviewed by our team ${ }^{[23,41]}$, and is driven by multiple signals including Wnts, high glucose, hypoxia, and EMT inducers. Historically, CBP and p300 have been considered largely redundant due to their significant protein sequence identity and even higher similarity. However, CBP and p300 are clearly not redundant and carry out definitive and unique roles both in vitro and in vivo ${ }^{[23,98-101]}$. From a library of 5000 secondary structure mimetics, we identified ICG-001 $\left(\mathrm{IC}_{50}=3 \mu \mathrm{M}\right)$ in a Wnt reporter screen in colon cancer cells. We subsequently identified and validated that the molecular target of ICG-001 was CBP and that ICG-001 binds specifically and with high affinity $(\sim 1 \mathrm{nM})$ to the N-terminus of CBP but not to $\mathrm{p} 300^{[102,103]}$. We subsequently found that selectively blocking the CBP/catenin interaction with ICG001, with an increase in p300/catenin-mediated transcription leads to the initiation of differentiation in stem and progenitor cells including ES, iPS, SSC and CSC [Figure $3 \mathrm{~A}]^{[104-108]}$. These investigations allowed us to propose our model of differential coactivator usage. The critical non-redundant roles that CBP and p300 play in catenin-mediated transcription are highlighted in our model ${ }^{[109]}$ [Figure 3B]. The model posits that catenin's choice to utilize either CBP or p300 is the first decision that guides a stem cell to either maintain 
A

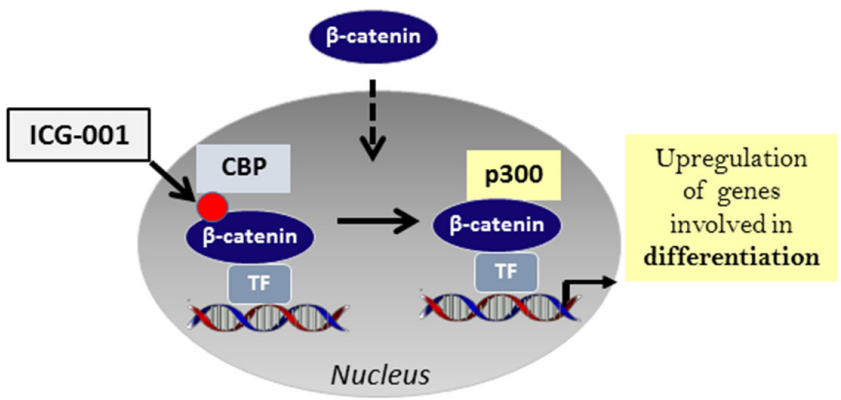

B

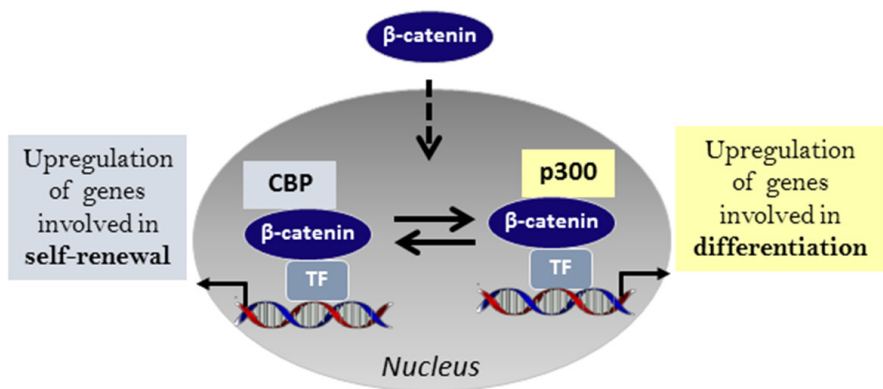

C

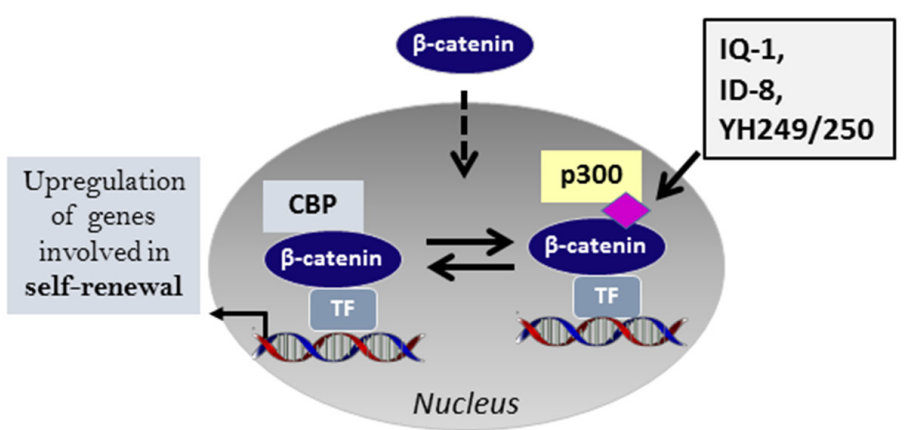

Figure 3. Differential Kat3 coactivator usage. A: ICG-001 specifically disrupts the interaction between CBP and $\beta$-catenin. This leads to increased $p 300 / \beta$-catenin transcription, a loss of the capacity to self-renew and the initiation of differentiation; B: $\beta$-catenin differential coactivator usage regulates differentiation versus self-renewal. $\beta$-catenin usage of either CBP or p300 leads to transcriptional activation of genes that are critical for self-renewal or differentiation respectively; C: IQ-1, ID8 (indirectly), and YH 249/250 (directly) disrupt the p300/ $\beta$-catenin interaction. Selectively antagonizing the $\mathrm{p} 300 / \beta$-catenin interaction enhances $\mathrm{CBP} / \beta$-catenin transcription thereby favoring self-renewal

potency or initiate a differentiative transcriptional program, respectively [Figure 3B]. We subsequently identified several small molecules, IQ-1 and ID-8, which are indirect p300/catenin inhibitors as well as the specific direct p300/catenin antagonists YH249/250. P300/catenin antagonists maintain the potency (plurior multipotency) of both mouse and human embryonic, induced pluripotent and somatic stem cells, by increasing CBP/catenin driven symmetric divisions both in vitro and in vivo ${ }^{[107,109-112]}$ [Figure 3C].

We have extensively examined the therapeutic potential of selectively antagonizing the $\mathrm{CBP} /$ catenin interaction, and have demonstrated the ability to safely eliminate drug-resistant CSC, via forced differentiation, without deleterious effects on the normal endogenous stem cell populations ${ }^{[104,105,113-115]}$. $\mathrm{CBP} /$ catenin antagonists can activate SSC and induce asymmetric differentiation thereby enhancing repair pathways in preclinical models of pulmonary and renal fibrosis ${ }^{[116,117]}$, myocardial infarction ${ }^{[118]}$ and neurodegeneration ${ }^{[23,108,119]}$. The differential effects of CBP/catenin antagonists on CSC versus SSC, specifically forced differentiation and elimination versus differentiation and enhanced repair without depletion, are cell intrinsic. CBP/catenin antagonists utilize the intrinsic propensity of CSC to preferentially divide symmetrically ${ }^{[36,37]}$ thereby stochastically eliminating CSC via forced symmetric divisions [Figure 4A]. 


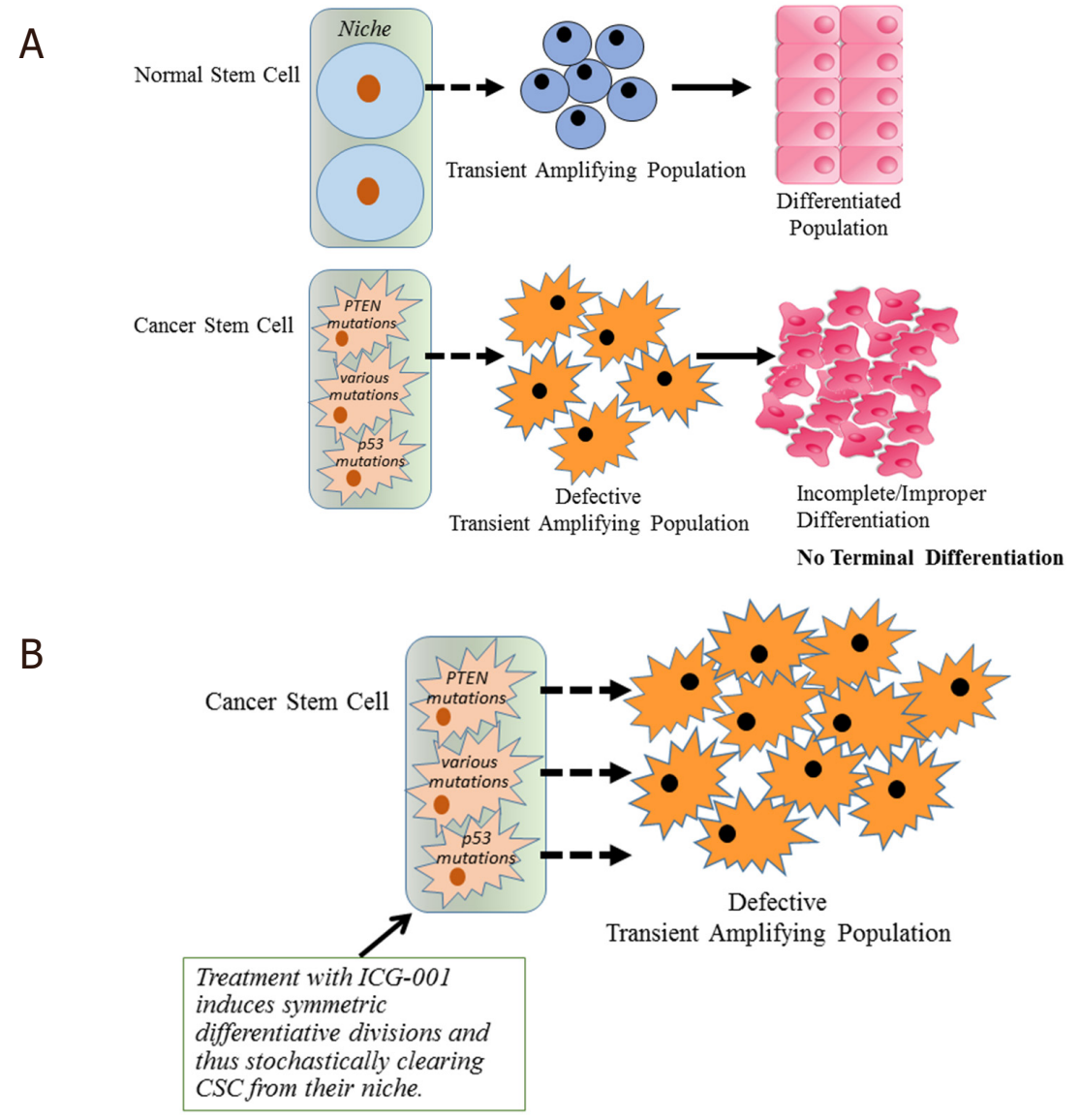

Figure 4. Intrinsic differences in the mode of division of SSC and CSC allow for the safe elimination of CSC via symmetric differentiative divisions. A: Asymmetric division is preferred in normal somatic stem cells (SSCs). Both symmetric and asymmetric divisions occur in cancer stem cells (CSC), thereby leading to an increase in the CSC population; B: CBP/catenin antagonists (e.g., ICG- 001) force symmetric differentiative divisions in CSC thereby driving the CSC population out of their niche. CBP/catenin antagonists maintain SSC asymmetric divisions thereby never depleting the niche

SSC preferentially differentiate asymmetrically, with one daughter cell always remaining in the niche and therefore are not depleted [Figure $4 \mathrm{~A}]^{[50]}$. Asymmetric differentiation can be activated by $\mathrm{CBP} / \mathrm{catenin}$ antagonists thereby enhancing repair without damaging the normal SSC population ${ }^{[23]}$. Therefore, CSC when treated with $\mathrm{CBP} /$ catenin antagonists will stochastically be cleared from their niche via symmetric differentiative divisions [Figure $4 \mathrm{~B}$ ].

Significant concerns about specificity arise when targeting the coactivator protein CBP, as it has as many as 500 molecular partners, including a vast array of transcription factors ${ }^{[119]}$. It is important to note that neither pre-clinical nor clinical studies have shown toxicity when utilizing specific small molecule $\mathrm{CBP} /$ catenin antagonists are safe. PRI-724 $\left(\mathrm{IC}_{50} \sim 150 \mathrm{nM}\right)$, a second-generation clinical CBP/catenin antagonist demonstrated an excellent safety profile in preclinical IND enabling toxicology studies. The no-adverse-event-level for PRI-724 in dogs was $120 \mathrm{mg} / \mathrm{kg} /$ day administered for 28-day via continuous infusion ${ }^{[120]}$. Clinically, PRI-724 had an excellent safety profile, demonstrating no dose limiting toxicities with escalation from 40 to $1280 \mathrm{mg} / \mathrm{m}^{2} /$ day administered by continuous i.v. infusion. Down regulation of the biomarker survivin/BIRC5 with upregulation of the differentiation antigen CK20 in EpCAM selected circulating tumor cells strongly correlated with increasing plasma concentrations of drug ${ }^{[120]}$. PRI-724 also demonstrated safety and efficacy with increased liver function in a trial conducted in patients with HCVinduced hepatic fibrosis ${ }^{[121]}$.This degree of safety was initially surprising. We believe this is due to the high 

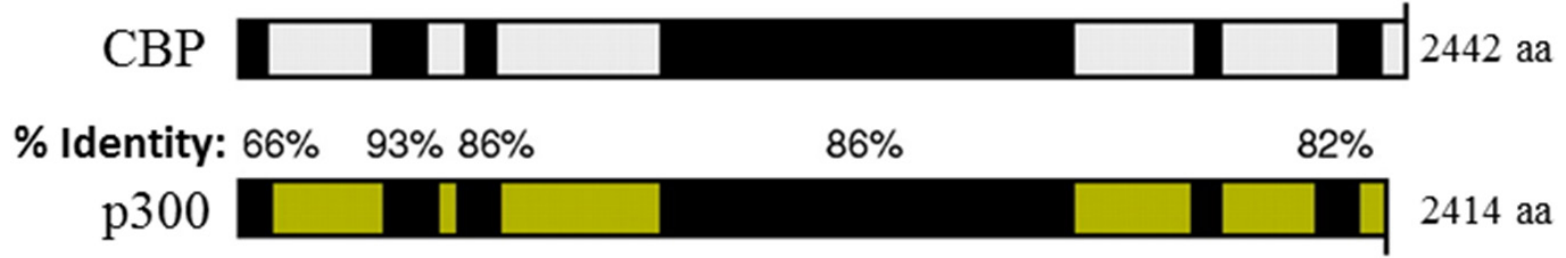

Figure 5. Despite having diverged more than 450 million years ago, CBP and $p 300$ possess a very high percentage of identity and even higher homology at the amino acid level. The most divergent region by far is the very amino termini of CBP and p300 to which ICG-001/ PRI-724 and YH249/250 bind respectively

biochemical specificity of ICG-001/PRI-724 for binding to CBP, and its limited impact on only a fraction of all CBP interactions. The unique non-redundant roles that the N-termini of the two Kat3 coactivators CBP and p300 play in stem cell biology and the intrinsic preference for asymmetric division in normal SSC are critical to the safety of these agents.

\section{Kat3A/CBP and Kat3B/p300 and SSC Metabolism}

"Nothing in Biology Makes Sense Except in the Light of Evolution" - Theodosius Dobzhansky.

Quiescence provides safeguards the functionality of SSC by restricting the damage caused by mitochondrial respiration and reactive oxygen species generated during oxidative phosphorylation. These safeguards limit DNA mutations and prevent uncontrolled cell cycle entry ${ }^{[122,123]}$. SSC and CSC preferentially utilize glycolysis over oxidative phosphorylation despite the inefficiency in regards to ATP generation of glycolysis compared to oxidative phosphorylation ${ }^{[124]}$. The activation of quiescent SSC and the initiation of differentiation involves a metabolic change from glycolysis and entry into the Krebs cycle. Reprogramming to pluripotency, on the other hand is associated with "anaerobicizing", ${ }^{,[25]}$. With the dawn of the evolution of vertebrates, roughly 450 million years ago, a new lifestyle having a relatively long-lived adult stage began. To accommodate this successfully a mechanism for long term homeostatic maintenance and tissue repair was essential. This was accomplished via quiescent "immortal" SSC maintaining an "anaerobic" metabolic state in specialized niches as opposed to their more proliferative aerobic-differentiated daughter cells. This mechanism evolved in order to protect genetic material integrity in long lived vertebrates ${ }^{[126]}$. Maintaining the two different populations resulting from asymmetric division; one daughter being a long-lived quiescent SSC utilizing anaerobic metabolism and the other a rapidly expanding differentiating population utilizing aerobic metabolism, required tight regulation. The Kat3 coactivator family CBP and p300 diverged via gene duplication just prior to the vertebrate radiation over 450 million years ago ${ }^{[127]}$. CBP and p300 are extremely large proteins encoded over 33 and 31 exons respectively. CBP and p300 retain extremely high identity, up to $93 \%$, particularly over a large central core that includes the $\mathrm{CH} 1, \mathrm{KIX}$, Bromodomain, and $\mathrm{CH} 2$ and $\mathrm{CH} 3$ regions [Figure 5], despite diverging over 450 million years ago ${ }^{[128,129]}$.

The small molecules CBP/catenin antagonists, ICG-001/PRI-724, and p300/catenin antagonists, YH $249 / 250$, bind the CBP and p300 N-termini, respectively ${ }^{[23,102,111,130]}$. This least conserved region between the two coactivators, which has only $66 \%$ identity, binds both $\beta$-catenin and through a highly conserved LXXLL sequence, nuclear receptor family members ${ }^{[131]}$. The $\mathrm{N}$-termini within each orthologous coactivator are extremely conserved with human and mouse CBP being 98\% identical at the amino acid level within this region. The nuclear receptor family and Wnt signaling appeared significantly earlier in evolution approximately 600 million years ago in the first multicellular animals (metazoans) ${ }^{[132,133]}$ and are found in nematodes, flies, and vertebrates.

Previously, we proposed that gene duplication generated the two Kat3 coactivators and a subsequent rapid divergence within their $\mathrm{N}$-terminal regions occurred at the same time as the integration of Wnt and nuclear 
receptor family signaling ${ }^{[23]}$. This co-evolution resulted in high fidelity control over the differential cell fates generated by asymmetric stem cell division, thereby enabling two inherently different cell populations and providing the expanding daughter cell population integrated pathways to generate divergent cell types. This joint genetic divergence and signaling integration additionally provided a mechanism to "read" multiple signals affecting SSC quiescence and DNA integrity. For example, a huge number of lesions in DNA can be induced by ultraviolet light ${ }^{[134]}$.Therefore, circadian regulation of normal SSC activation and division is critical $^{[135]}$. Shift workers, with aberrant circadian regulation, have an increased risk for the development of cancer ${ }^{[136,137]}$. Metabolic and circadian regulation control the timing and the mode of SSC division ${ }^{[138]}$ and metabolic pathways through nuclear receptors (e.g., PPAR, Rev-Erb $\alpha$ and $\beta$ ) play critical roles in circadian integration of metabolism energetics ${ }^{[139,140]}$. Clock, part of the master circadian regulatory circuit mediated by the Clock/Bmal1 transcriptional complex, is recruited to p300 in vivo in a time-dependent manner ${ }^{[141]}$. Evolutionarily, it would seem logical that mechanisms to enhance SSC asymmetric differentiation and symmetric differentiative divisions of CSC or pre-CSC would have evolved. In fact, numerous naturally occurring $\mathrm{CBP} /$ catenin antagonists have evolved. Returning to the concept of differentiation therapy, alltrans retinoic acid (ATRA), a vitamin A derivative, via its nuclear receptor complex (RAR/RXR) acting as a $\mathrm{CBP} /$ catenin antagonist is very effective in treating Acute Promyelocytic Leukemia. ATRA, similar to ICG001 does not kill malignant cells but rather induces them to differentiate. Vitamin D plays an important role in cancer prevention through the (VDR/RXR) nuclear receptor complex and both ATRA and VitD have been shown to antagonize aberrant Wnt signaling in the context of malignancy ${ }^{[142]}$. Nuclear receptor family members, via competition with $\beta$-catenin for binding to the $\mathrm{N}$-terminus of $\mathrm{CBP}$, phenocopy $\mathrm{CBP} /$ catenin antagonists. However, synergistic effects on the activation of gene expression by nuclear receptors and Wnt signaling have been demonstrated (e.g., ATRA and Wnt) ${ }^{[143]}$ and nuclear receptors also on their own control the expression of various transcriptional cassettes. Thus, nuclear receptor family members are not simply "pure antagonists" of $\mathrm{CBP} /$ catenin transcription and therefore have significant differences from small molecule direct $\mathrm{CBP} /$ catenin antagonists.

The LXXLL sequence present in the amino termini of both CBP and p300 is highly conserved and can recruit both RAR/RXR and VDR/RXR complexes, and potentially all other nuclear receptor complexes including AR, PPAR, and others. Not surprisingly, multiple nuclear receptors can effect stem cell maintenance or initiate differentiation in a manner similar to small molecule p300/catenin or $\mathrm{CBP} /$ catenin antagonists $^{[23,144]}$. However, in contrast to modulation of nuclear receptors, which can cause developmental defects, selectively antagonizing the CBP/catenin interaction with ICG-001, even at very high levels, is extremely safe and has no deleterious effects on mouse embryonic development ${ }^{[118,145]}$. Female mice treated topically or orally with high doses of ICG-001 throughout pregnancy have normal litters. The pups exhibited normal weight and size compared to their control littermates and can reproduce normally, demonstrating no deleterious effects to germ cell populations, which interestingly, also prefer asymmetric divisions $^{[146,147]}$. Interestingly, a $27 \mathrm{bp} / 9 \mathrm{aa}$ deletion in CBP between the $\beta$-catenin-binding region (DELIsequence) and the nuclear receptor (LXXLL) binding sequence is a strongly evolutionarily conserved. Using CRISPR/Cas9 editing of p300, we recently demonstrated that this deletion in CBP provided a mechanism via steric inhibition, for nuclear receptors to antagonize $\mathrm{CBP} /$ catenin signaling, allowing for the maintenance of quiescence and initiation of asymmetric divisions in SSC. Whereas $\beta$-catenin and nuclear receptor signaling can synergize to effect a feed-forward mechanism to drive differentiation and lineage commitment utilizing $\mathrm{p} 300$, as steric constraints removed by the conserved 9 amino acid insertion is sufficient to allow for the simultaneous binding of nuclear receptors and $\beta$-catenin ${ }^{[130]}$.

\section{Summary: CSC resistance and differential Kat3 coactivator usage}

SSC and CSC utilize Wnt/catenin signaling and differential Kat3 coactivator usage to regulate stem cell homeostasis and the balance between self-renewal and differentiation. The fundamental difference between SSC and CSC appears to be a preference for asymmetric over symmetric divisions respectively. Increased 
$\mathrm{CBP} /$ catenin transcription is associated with enhanced telomerase activity and the expression of BIRC5/ Survivin ${ }^{[98]}$ required for self-renewal of stem cells. In this regard, targeting CBP/catenin signaling appears to represent a common "Achilles' Heel" in CSC in both solid and liquid tumors ${ }^{[23,104,113,148]}$. Aberrant regulation of catenin/Kat3 coactivator usage enhances CBP/catenin activation at the expense of p300/cateninmediated transcription. Preferential use of this coactivator can arise from a vast array of mutations, either inherited or acquired, and a wide variety of insults (i.e., chronic inflammation, viral infection, high fat/ caloric diet, and others). Resistance to therapy, radiation, chemotherapy of immunotherapy is associated with selection of resistant clone(s) from a pre-existing CSC pool. CBP/catenin antagonists, by taking advantage of this fundamental difference between SSC and CSC can safely stochastically differentiate away symmetrically dividing CSC without depleting the SSC population that is dividing symmetrically. However, in cancer, the transient amplifying population is not sensitive to $\mathrm{CBP} /$ catenin antagonists and still must be targeted to eliminate the disease, as these populations rely on other pathways (Bcr-Abl, KRAS, etc.) to maintain their non-terminally differentiated proliferative status ${ }^{[104,105,114]}$. The robust safety profile of $\mathrm{CBP} /$ catenin antagonists could eventually provide an opportunity to utilize them in a "vitamin-like" manner as a prophylaxis to the accumulation of pre-CSC or CSCs.

\section{DECLARATIONS}

\section{Authors' contributions}

Funded the studies, writing and final approval of the Perspective: Kahn M

Contributed additional concepts, writing and edited manuscript: Bild A, Teo JL

\section{Availability of data and materials}

Not applicable.

\section{Financial support and sponsorship}

Kahn M has been supported by NIH P30CA014089, R01CA166161, R21NS074392, R21AI105057, and R01HL112638. Bild A has been supported by NIH U54CA209978.

\section{Conflicts of interest}

All authors declared that there are no conflicts of interest.

\section{Ethical approval and consent to participate}

Not applicable.

\section{Consent for publication}

Not applicable.

\section{Copyright}

(c) The Author(s) 2019.

\section{REFERENCES}

1. Global Burden of Disease Cancer C, Fitzmaurice C, Akinyemiju TF, Al Lami FH, Alam T, et al. Global, regional, and national cancer incidence, mortality, years of life lost, years lived with disability, and disability-adjusted life-years for 29 cancer groups, 1990 to 2016 : a systematic analysis for the global burden of disease study. JAMA Oncol 2018;4:1553-68.

2. Sabnis AJ, Bivona TG. Principles of resistance to targeted cancer therapy: lessons from basic and translational cancer biology. Trends Mol Med 2019;25:185-97.

3. Reya T, Morrison SJ, Clarke MF, Weissman IL. Stem cells, cancer, and cancer stem cells. Nature 2001;414:105-11.

4. Yadav AK, Desai NS. Cancer stem cells: acquisition, characteristics, therapeutic implications, targeting strategies and future prospects. Stem Cell Rev 2019;15:331-55. 
5. Agnoletto C, Corra F, Minotti L, Baldassari F, Crudele F, et al. Heterogeneity in circulating tumor cells: the relevance of the stem-cell subset. Cancers (Basel) 2019;11.

6. Afify SM, Seno M. Conversion of stem cells to cancer stem cells: undercurrent of cancer initiation. Cancers (Basel) 2019;11.

7. Batlle E, Clevers H. Cancer stem cells revisited. Nat Med 2017;23:1124-34.

8. Ramos EK, Hoffmann AD, Gerson SL, Liu H. New opportunities and challenges to defeat cancer stem cells. Trends Cancer 2017;3:780-96.

9. Cohnheim J. Ueber entzündung und Eiterung. Path Anat Physiol Klin Med. 1867;40:1-79.

10. Holland JD, Gyorffy B, Vogel R, Eckert K, Valenti G, et al. Combined Wnt/beta-catenin, Met, and CXCL12/CXCR4 signals characterize basal breast cancer and predict disease outcome. Cell Rep 2013;5:1214-27.

11. Liu S, Wicha MS. Targeting breast cancer stem cells. J Clin Oncol 2010;28:4006-12.

12. Vassiliou GS, Cooper JL, Rad R, Li J, Rice S, et al. Mutant nucleophosmin and cooperating pathways drive leukemia initiation and progression in mice. Nat Genet 2011;43:470-5.

13. Gudjonsson T, Magnusson MK. Stem cell biology and the cellular pathways of carcinogenesis. APMIS 2005;113:922-9.

14. Al-Hajj M, Clarke MF. Self-renewal and solid tumor stem cells. Oncogene 2004;23:7274-82.

15. Chaffer CL, Brueckmann I, Scheel C, Kaestli AJ, Wiggins PA, et al. Normal and neoplastic nonstem cells can spontaneously convert to a stem-like state. Proc Natl Acad Sci U S A 2011;108:7950-5.

16. Wainwright EN, Scaffidi P. Epigenetics and cancer stem cells: unleashing, hijacking, and restricting cellular plasticity. Trends Cancer 2017;3:372-86.

17. Dalerba P, Dylla SJ, Park I-K, Liu R, Wang X, et al. Phenotypic characterization of human colorectal cancer stem cells. Proc Natl Acad Sci U S A 2007;104:10158-63.

18. Dean M, Fojo T, Bates S. Tumour stem cells and drug resistance. Nat Rev Cancer 2005;5:275-84.

19. Bleau AM, Hambardzumyan D, Ozawa T, Fomchenko EI, Huse JT, et al. PTEN/PI3K/Akt pathway regulates the side population phenotype and ABCG2 activity in glioma tumor stem-like cells. Cell Stem Cell 2009;4:226-35.

20. Nieborowska-Skorska M, Sullivan K, Dasgupta Y, Podszywalow-Bartnicka P, Hoser G, et al. Gene expression and mutation-guided synthetic lethality eradicates proliferating and quiescent leukemia cells. J Clin Invest 2017;127:2392-406.

21. Thiery JP. Epithelial-mesenchymal transitions in tumour progression. Nat Rev Cancer 2002;2:442-54.

22. Croucher PI, McDonald MM, Martin TJ. Bone metastasis: the importance of the neighbourhood. Nat Rev Cancer 2016;16:373-86.

23. Thomas PD, Kahn M. Kat3 coactivators in somatic stem cells and cancer stem cells: biological roles, evolution, and pharmacologic manipulation. Cell Biol Toxicol 2016;32:61-81.

24. Cullen SM, Mayle A, Rossi L, Goodell MA. Hematopoietic stem cell development: an epigenetic journey. Curr Top Dev Biol 2014;107:39-75.

25. Gage FH, Temple S. Neural stem cells: generating and regenerating the brain. Neuron 2013;80:588-601.

26. Clevers H. The intestinal crypt, a prototype stem cell compartment. Cell 2013;154:274-84.

27. LaBarge MA. The difficulty of targeting cancer stem cell niches. Clin Cancer Res 2010;16:3121-9.

28. Merchant AA, Matsui W. Targeting Hedgehog-a cancer stem cell pathway. Clin Cancer Res 2010;16:3130-40.

29. Pannuti A, Foreman K, Rizzo P, Osipo C, Golde T, et al. Targeting Notch to target cancer stem cells. Clin Cancer Res 2010;16:3141-52.

30. Saygin C, Matei D, Majeti R, Reizes O, Lathia JD. Targeting cancer stemness in the clinic: from hype to hope. Cell Stem Cell 2019;24:25-40.

31. Crupi MJF, Bell JC, Singaravelu R. Concise review: targeting cancer stem cells and their supporting niche using oncolytic viruses. Stem Cells 2019;37:716-23.

32. Luo M, Wicha MS. Targeting cancer stem cell redox metabolism to enhance therapy responses. Semin Radiat Oncol 2019;29:42-54.

33. Foudi A, Hochedlinger K, Van Buren D, Schindler JW, Jaenisch R, et al. Analysis of histone 2B-GFP retention reveals slowly cycling hematopoietic stem cells. Nat Biotechnol 2009;27:84-90.

34. Baker AM, Cereser B, Melton S, Fletcher AG, Rodriguez-Justo M, et al. Quantification of crypt and stem cell evolution in the normal and neoplastic human colon. Cell Rep 2014;8:940-7.

35. Cairns J. Mutation selection and the natural history of cancer. Nature 1975;255:197-200.

36. Cicalese A, Bonizzi G, Pasi CE, Faretta M, Ronzoni S, et al. The tumor suppressor p53 regulates polarity of self-renewing divisions in mammary stem cells. Cell 2009;138:1083-95.

37. Lee JY, Nakada D, Yilmaz OH, Tothova Z, Joseph NM, et al. mTOR activation induces tumor suppressors that inhibit leukemogenesis and deplete hematopoietic stem cells after Pten deletion. Cell Stem Cell 2010;7:593-605.

38. Ferent J, Cochard L, Faure H, Taddei M, Hahn H, et al. Genetic activation of hedgehog signaling unbalances the rate of neural stem cell renewal by increasing symmetric divisions. Stem Cell Reports 2014;3:312-23.

39. Arends CM, Galan-Sousa J, Hoyer K, Chan W, Jager M, et al. Hematopoietic lineage distribution and evolutionary dynamics of clonal hematopoiesis. Leukemia 2018;32:1908-19.

40. Jaiswal S, Natarajan P, Silver AJ, Gibson CJ, Bick AG, et al. Clonal hematopoiesis and risk of atherosclerotic cardiovascular disease. N Engl J Med 2017;377:111-21.

41. Kahn M. Can we safely target the WNT pathway? Nat Rev Drug Discov 2014;13:513-32.

42. Niehrs C. The complex world of WNT receptor signalling. Nat Rev Mol Cell Biol 2012;13:767-79.

43. Moon RT. Wnt/beta-catenin pathway. Sci STKE 2005;2005:cm1.

44. Lai KKY, Nguyen C, Lee KS, Lee A, Lin DP, et al. Convergence of canonical and non-canonical Wnt signal: differential Kat3 
coactivator usage. Curr Mol Pharmacol 2019; [Epub ahead of print].

45. Kim YM, Ma H, Oehler VG, Gang EJ, Nguyen C, et al. The gamma catenin/CBP complex maintains survivin transcription in betacatenin deficient/depleted cancer cells. Curr Cancer Drug Targets 2011;11:213-25.

46. Kim K, Daniels KJ, Hay ED. Tissue-specific expression of beta-catenin in normal mesenchyme and uveal melanomas and its effect on invasiveness. Exp Cell Res 1998;245:79-90.

47. Onder TT, Gupta PB, Mani SA, Yang J, Lander ES, et al. Loss of E-cadherin promotes metastasis via multiple downstream transcriptional pathways. Cancer Res 2008;68:3645-54.

48. Wagh PK, Gray JK, Zinser GM, Vasiliauskas J, James L, et al. beta-Catenin is required for Ron receptor-induced mammary tumorigenesis. Oncogene 2011;30:3694-704.

49. Coluccia AM, Benati D, Dekhil H, De Filippo A, Lan C, et al. SKI-606 decreases growth and motility of colorectal cancer cells by preventing pp60(c-Src)-dependent tyrosine phosphorylation of beta-catenin and its nuclear signaling. Cancer Res 2006;66:2279-86.

50. Ress A, Moelling K. Bcr interferes with beta-catenin-Tcf1 interaction. FEBS Lett 2006;580:1227-30.

51. Ishimoto T, Oshima H, Oshima M, Kai K, Torii R, et al. CD44+ slow-cycling tumor cell expansion is triggered by cooperative actions of Wnt and prostaglandin E2 in gastric tumorigenesis. Cancer Sci 2010;101:673-8.

52. Kida A, Kahn M. Hypoxia selects for a quiescent, CML stem/leukemia initiating-like population dependent on $\mathrm{CBP} / \mathrm{catenin}$ transcription. Curr Mol Pharmacol 2013;6:204-10.

53. Mazumdar J, O’Brien WT, Johnson RS, LaManna JC, Chavez JC, et al. O2 regulates stem cells through Wnt/beta-catenin signalling. Nat Cell Biol 2010;12:1007-13.

54. Chocarro-Calvo A, Garcia-Martinez JM, Ardila-Gonzalez S, De la Vieja A, Garcia-Jimenez C. Glucose-induced beta-catenin acetylation enhances Wnt signaling in cancer. Mol Cell 2013;49:474-86.

55. Zhao CM, Hayakawa Y, Kodama Y, Muthupalani S, Westphalen CB, et al. Denervation suppresses gastric tumorigenesis. Sci Transl Med 2014;6:250ra115.

56. Kawabata A. Prostaglandin E2 and pain-an update. Biol Pharm Bull 2011;34:1170-3.

57. van Veelen W, Le NH, Helvensteijn W, Blonden L, Theeuwes M, et al. $\beta$-catenin tyrosine 654 phosphorylation increases Wnt signalling and intestinal tumorigenesis. Gut 2011;60:1204-12.

58. Brembeck FH, Rosario M, Birchmeier W. Balancing cell adhesion and Wnt signaling, the key role of beta-catenin. Curr Opin Genet Dev 2006;16:51-9.

59. Komiya Y, Habas R. Wnt signal transduction pathways. Organogenesis 2008;4:68-75.

60. Sato N, Meijer L, Skaltsounis L, Greengard P, Brivanlou AH. Maintenance of pluripotency in human and mouse embryonic stem cells through activation of Wnt signaling by a pharmacological GSK-3-specific inhibitor. Nat Med 2004;10:55-63.

61. Otero JJ, Fu W, Kan L, Cuadra AE, Kessler JA. Beta-catenin signaling is required for neural differentiation of embryonic stem cells. Development 2004;131:3545-57.

62. Kahn M. Symmetric division versus asymmetric division: a tale of two coactivators. Future Med Chem 2011;3:1745-63.

63. Kuhl SJ, Kuhl M. On the role of Wnt/beta-catenin signaling in stem cells. Biochim Biophys Acta 2013;1830:2297-306.

64. Anastas JN, Moon RT. WNT signalling pathways as therapeutic targets in cancer. Nat Rev Cancer 2013;13:11-26.

65. Polakis P. Wnt signaling in cancer. Cold Spring Harb Perspect Biol 2012;4.

66. Blum B, Bar-Nur O, Golan-Lev T, Benvenisty N. The anti-apoptotic gene survivin contributes to teratoma formation by human embryonic stem cells. Nat Biotechnol 2009;27:281-7.

67. Hoffmeyer K, Raggioli A, Rudloff S, Anton R, Hierholzer A, et al. Wnt/beta-catenin signaling regulates telomerase in stem cells and cancer cells. Science 2012;336:1549-54.

68. DiMeo TA, Anderson K, Phadke P, Fan C, Perou CM, et al. A novel lung metastasis signature links Wnt signaling with cancer cell self-renewal and epithelial-mesenchymal transition in basal-like breast cancer. Cancer Res 2009;69:5364-73.

69. Mani SA, Guo W, Liao MJ, Eaton EN, Ayyanan A, et al. The epithelial-mesenchymal transition generates cells with properties of stem cells. Cell 2008;133:704-15.

70. Sack U, Walther W, Scudiero D, Selby M, Aumann J, et al. S100A4-induced cell motility and metastasis is restricted by the Wnt/betacatenin pathway inhibitor calcimycin in colon cancer cells. Mol Biol Cell 2011;22:3344-54.

71. Yu M, Ting DT, Stott SL, Wittner BS, Ozsolak F, et al. RNA sequencing of pancreatic circulating tumour cells implicates WNT signalling in metastasis. Nature 2012;487:510-3.

72. Zhuang X, Zhang H, Li X, Li X, Cong M, et al. Differential effects on lung and bone metastasis of breast cancer by Wnt signalling inhibitor DKK1. Nat Cell Biol 2017;19:1274-85.

73. Peinado H, Zhang H, Matei IR, Costa-Silva B, Hoshino A, et al. Pre-metastatic niches: organ-specific homes for metastases. Nat Rev Cancer 2017;17:302-17.

74. Lickert H, Domon C, Huls G, Wehrle C, Duluc I, et al. Wnt/(beta)-catenin signaling regulates the expression of the homeobox gene Cdx1 in embryonic intestine. Development 2000;127:3805-13.

75. Russell RG, Lasorella A, Dettin LE, Iavarone A. Id2 drives differentiation and suppresses tumor formation in the intestinal epithelium. Cancer Res 2004;64:7220-5.

76. Barker N, van Es JH, Kuipers J, Kujala P, van den Born M, et al. Identification of stem cells in small intestine and colon by marker gene Lgr5. Nature 2007;449:1003-7.

77. Wielenga VJ, Smits R, Korinek V, Smit L, Kielman M, et al. Expression of CD44 in Apc and Tcf mutant mice implies regulation by the WNT pathway. Am J Pathol 1999;154:515-23. 
78. Shulewitz M, Soloviev I, Wu T, Koeppen H, Polakis P, et al. Repressor roles for TCF-4 and Sfrp1 in Wnt signaling in breast cancer. Oncogene 2006;25:4361-9.

79. Katoh Y, Katoh M. Comparative genomics on PROM1 gene encoding stem cell marker CD133. Int J Mol Med 2007;19:967-70.

80. Correa S, Binato R, Du Rocher B, Castelo-Branco MT, Pizzatti L, et al. Wnt/beta-catenin pathway regulates ABCB1 transcription in chronic myeloid leukemia. BMC Cancer 2012;12:303.

81. Yamada T, Takaoka AS, Naishiro Y, Hayashi R, Maruyama K, et al. Transactivation of the multidrug resistance 1 gene by T-cell factor 4/beta-catenin complex in early colorectal carcinogenesis. Cancer Res 2000;60:4761-6.

82. Yamashita T, Budhu A, Forgues M, Wang XW. Activation of hepatic stem cell marker EpCAM by Wnt-beta-catenin signaling in hepatocellular carcinoma. Cancer Res 2007;67:10831-9.

83. Al-Hajj M, Wicha MS, Benito-Hernandez A, Morrison SJ, Clarke MF. Prospective identification of tumorigenic breast cancer cells. Proc Natl Acad Sci U S A 2003;100:3983-8.

84. Wang Y, Krivtsov AV, Sinha AU, North TE, Goessling W, et al. The Wnt/beta-catenin pathway is required for the development of leukemia stem cells in AML. Science 2010;327:1650-3.

85. Radich JP, Dai H, Mao M, Oehler V, Schelter J, et al. Gene expression changes associated with progression and response in chronic myeloid leukemia. Proc Natl Acad Sci U S A 2006;103:2794-9.

86. Pehlivan M, Sercan Z, Sercan HO. sFRP1 promoter methylation is associated with persistent Philadelphia chromosome in chronic myeloid leukemia. Leuk Res 2009;33:1062-7.

87. Zhao Y, Tao L, Yi J, Song H, Chen L. The role of canonical Wnt signaling in regulating radioresistance. Cell Physiol Biochem 2018;48:419-32.

88. Katoh M. Canonical and non-canonical WNT signaling in cancer stem cells and their niches: cellular heterogeneity, omics reprogramming, targeted therapy and tumor plasticity (Review). Int J Oncol 2017;51:1357-69.

89. Spranger S, Gajewski TF. Impact of oncogenic pathways on evasion of antitumour immune responses. Nat Rev Cancer 2018;18:139-47.

90. Schinzari V, Timperi E, Pecora G, Palmucci F, Gallerano D, et al. Wnt3a/beta-catenin signaling conditions differentiation of partially exhausted T-effector cells in human cancers. Cancer Immunol Res 2018;6:941-52.

91. Suryawanshi A, Manicassamy S. Tumors induce immune tolerance through activation of beta-catenin/TCF4 signaling in dendritic cells: A novel therapeutic target for cancer immunotherapy. Oncoimmunology 2015;4:e1052932.

92. Wang B, Tian T, Kalland KH, Ke X, Qu Y. Targeting Wnt/beta-catenin signaling for cancer immunotherapy. Trends Pharmacol Sci 2018;39:648-58.

93. Takahashi-Yanaga F, Kahn M. Targeting Wnt signaling: can we safely eradicate cancer stem cells? Clin Cancer Res 2010;16:3153-62.

94. Acebron SP, Niehrs C. beta-catenin-independent roles of Wnt/LRP6 signaling. Trends Cell Biol 2016;26:956-67.

95. Veeman MT, Axelrod JD, Moon RT. A second canon. Functions and mechanisms of beta-catenin-independent Wnt signaling. Dev Cell 2003;5:367-77.

96. Nowak D, Stewart D, Koeffler HP. Differentiation therapy of leukemia: 3 decades of development. Blood 2009;113:3655-65.

97. Teo JL, Kahn M. The Wnt signaling pathway in cellular proliferation and differentiation: A tale of two coactivators. Adv Drug Deliv Rev 2010;62:1149-55.

98. Ma H, Nguyen C, Lee KS, Kahn M. Differential roles for the coactivators CBP and p300 on TCF/beta-catenin-mediated survivin gene expression. Oncogene 2005;24:3619-31.

99. Roth JF, Shikama N, Henzen C, Desbaillets I, Lutz W, et al. Differential role of p300 and CBP acetyltransferase during myogenesis: p300 acts upstream of MyoD and Myf5. EMBO J 2003;22:5186-96.

100. Yamauchi T, Oike Y, Kamon J, Waki H, Komeda K, et al. Increased insulin sensitivity despite lipodystrophy in Crebbp heterozygous mice. Nat Genet 2002;30:221-6.

101. Kung AL, Rebel VI, Bronson RT, Ch'ng LE, Sieff CA, et al. Gene dose-dependent control of hematopoiesis and hematologic tumor suppression by CBP. Genes Dev 2000;14:272-7.

102. Emami KH, Nguyen C, Ma H, Kim DH, Jeong KW, et al. A small molecule inhibitor of beta-catenin/CREB-binding protein transcription [corrected]. Proc Natl Acad Sci U S A 2004;101:12682-7.

103. McMillan M, Kahn M. Investigating Wnt signaling: a chemogenomic safari. Drug Discov Today 2005;10:1467-74.

104. Manegold P, Lai KKY, Wu Y, Teo JL, Lenz HJ, et al. Differentiation therapy targeting the beta-catenin/CBP interaction in pancreatic cancer. Cancers (Basel) 2018;10.

105. Zhao Y, Masiello D, McMillian M, Nguyen C, Wu Y, et al. CBP/catenin antagonist safely eliminates drug-resistant leukemia-initiating cells. Oncogene 2016;35:3705-17.

106. Banerjee ER, Laflamme MA, Papayannopoulou T, Kahn M, Murry CE, et al. Human embryonic stem cells differentiated to lung lineage-specific cells ameliorate pulmonary fibrosis in a xenograft transplant mouse model. PLoS One 2012;7:e33165.

107. Hasegawa K, Yasuda SY, Teo JL, Nguyen C, McMillan M, et al. Wnt signaling orchestration with a small molecule DYRK inhibitor provides long-term xeno-free human pluripotent cell expansion. Stem Cells Transl Med 2012;1:18-28.

108. Teo JL, Ma H, Nguyen C, Lam C, Kahn M. Specific inhibition of CBP/beta-catenin interaction rescues defects in neuronal differentiation caused by a presenilin-1 mutation. Proc Natl Acad Sci U S A 2005;102:12171-6.

109. Miyabayashi T, Teo JL, Yamamoto M, McMillan M, Nguyen C, et al. Wnt/beta-catenin/CBP signaling maintains long-term murine embryonic stem cell pluripotency. Proc Natl Acad Sci U S A 2007;104:5668-73.

110. Zhao Y, Wu K, Nguyen C, Smbatyan G, Melendez E, et al. Small molecule p300/catenin antagonist enhances hematopoietic recovery after radiation. PLoS One 2017;12:e0177245. 
111. Higuchi Y, Nguyen C, Yasuda SY, McMillan M, Hasegawa K, et al. Specific direct small molecule p300/beta-catenin antagonists maintain stem cell potency. Curr Mol Pharmacol 2016;9:272-9.

112. Marson A, Foreman R, Chevalier B, Bilodeau S, Kahn M, et al. Wnt signaling promotes reprogramming of somatic cells to pluripotency. Cell Stem Cell 2008;3:132-5.

113. Ring A, Nguyen C, Smbatyan G, Tripathy D, Yu M, et al. CBP/beta-catenin/FOXM1 is a novel therapeutic target in triple negative breast cancer. Cancers (Basel) 2018;10

114. Gang EJ, Hsieh YT, Pham J, Zhao Y, Nguyen C, et al. Small-molecule inhibition of CBP/catenin interactions eliminates drug-resistant clones in acute lymphoblastic leukemia. Oncogene 2014;33:2169-78.

115. Wend P, Fang L, Zhu Q, Schipper JH, Loddenkemper C, et al. Wnt/beta-catenin signalling induces MLL to create epigenetic changes in salivary gland tumours. EMBO J 2013;32:1977-89.

116. Hao S, He W, Li Y, Ding H, Hou Y, et al. Targeted inhibition of beta-catenin/CBP signaling ameliorates renal interstitial fibrosis. J Am Soc Nephrol 2011;22:1642-53.

117. Henderson WR, Jr., Chi EY, Ye X, Nguyen C, Tien YT, et al. Inhibition of Wnt/beta-catenin/CREB binding protein (CBP) signaling reverses pulmonary fibrosis. Proc Natl Acad Sci U S A 2010;107:14309-14.

118. Sasaki T, Hwang H, Nguyen C, Kloner RA, Kahn M. The small molecule Wnt signaling modulator ICG-001 improves contractile function in chronically infarcted rat myocardium. PLoS One 2013;8:e75010.

119. Ring A, Kim YM, Kahn M. Wnt/catenin signaling in adult stem cell physiology and disease. Stem Cell Rev 2014;10:512-25.

120. El-Khoueiry AB, Ning Y, Yang D, Cole S, Kahn M, et al. A phase I first-in-human study of PRI-724 in patients (pts) with advanced solid tumors. J Clin Oncol 2013;31:2501.

121. Kimura K, Ikoma A, Shibakawa M, Shimoda S, Harada K, et al. Safety, tolerability, and preliminary efficacy of the anti-fibrotic small molecule PRI-724, a CBP/ $\beta$-catenin inhibitor, in patients with hepatitis C virus-related cirrhosis: a single-center, open-label, dose escalation phase 1 trial. EBioMedicine 2017;23:79-87.

122. Bakker ST, Passegue E. Resilient and resourceful: genome maintenance strategies in hematopoietic stem cells. Exp Hematol 2013;41:915-23.

123. Orford KW, Scadden DT. Deconstructing stem cell self-renewal: genetic insights into cell-cycle regulation. Nat Rev Genet 2008;9:115-28.

124. Kohli L, Passegue E. Surviving change: the metabolic journey of hematopoietic stem cells. Trends Cell Biol 2014;24:479-87.

125. Panopoulos AD, Izpisua Belmonte JC. Anaerobicizing into pluripotency. Cell Metab 2011;14:143-4.

126. Trosko JE, Kang KS. Evolution of energy metabolism, stem cells and cancer stem cells: how the warburg and barker hypotheses might be linked. Int J Stem Cells 2012;5:39-56.

127. Mi H, Muruganujan A, Thomas PD. PANTHER in 2013: modeling the evolution of gene function, and other gene attributes, in the context of phylogenetic trees. Nucleic Acids Res 2013;41:D377-86.

128. Arany Z, Sellers WR, Livingston DM, Eckner R. E1A-associated p300 and CREB-associated CBP belong to a conserved family of coactivators. Cell 1994;77:799-800.

129. Eckner R, Arany Z, Ewen M, Sellers W, Livingston DM. The adenovirus E1A-associated 300-kD protein exhibits properties of a transcriptional coactivator and belongs to an evolutionarily conserved family. Cold Spring Harb Symp Quant Biol 1994;59:85-95.

130. Ono M, Lai KKY, Wu K, Nguyen C, Lin DP, et al. Nuclear receptor/Wnt beta-catenin interactions are regulated via differential CBP/ p300 coactivator usage. PLoS One 2018;13:e0200714.

131. Kamei Y, Xu L, Heinzel T, Torchia J, Kurokawa R, et al. A CBP integrator complex mediates transcriptional activation and AP-1 inhibition by nuclear receptors. Cell 1996;85:403-14.

132. Holstein TW. The evolution of the Wnt pathway. Cold Spring Harb Perspect Biol 2012;4:a007922.

133. Markov GV, Laudet V. Origin and evolution of the ligand-binding ability of nuclear receptors. Mol Cell Endocrinol 2011;334:21-30.

134. Jackson SP, Bartek J. The DNA-damage response in human biology and disease. Nature 2009;461:1071-8.

135. Brown SA. Circadian clock-mediated control of stem cell division and differentiation: beyond night and day. Development 2014;141:3105-11.

136. Davis S, Mirick DK, Stevens RG. Night shift work, light at night, and risk of breast cancer. J Natl Cancer Inst 2001;93:1557-62.

137. Schernhammer ES, Laden F, Speizer FE, Willett WC, Hunter DJ, et al. Rotating night shifts and risk of breast cancer in women participating in the nurses' health study. J Natl Cancer Inst 2001;93:1563-8.

138. Chen Z, McKnight SL. A conserved DNA damage response pathway responsible for coupling the cell division cycle to the circadian and metabolic cycles. Cell Cycle 2007;6:2906-12.

139. Dierickx P, Van Laake LW, Geijsen N. Circadian clocks: from stem cells to tissue homeostasis and regeneration. EMBO Rep 2018;19:18-28.

140. Bass J, Takahashi JS. Circadian integration of metabolism and energetics. Science 2010;330:1349-54.

141. Etchegaray JP, Lee C, Wade PA, Reppert SM. Rhythmic histone acetylation underlies transcription in the mammalian circadian clock. Nature 2003;421:177-82.

142. Dillard AC, Lane MA. Retinol decreases beta-catenin protein levels in retinoic acid-resistant colon cancer cell lines. Mol Carcinog 2007;46:315-29.

143. Szeto W, Jiang W, Tice DA, Rubinfeld B, Hollingshead PG, et al. Overexpression of the retinoic acid-responsive gene \&lt;strong\&gt ;\&lt;em\&gt;Stra6\&lt;/em\&gt;\&lt;/strong\&gt; in human cancers and its synergistic induction by Wnt-1 and retinoic acid. Cancer Res 2001;61:4197-205. 
144. Mullen EM, Gu P, Cooney AJ. Nuclear receptors in regulation of mouse ES cell pluripotency and differentiation. PPAR Res 2007;2007:61563.

145. Sasaki T, Kahn M. Inhibition of beta-catenin/p300 interaction proximalizes mouse embryonic lung epithelium. Transl Respir Med 2014;2:8.

146. Suzuki S, Nozawa Y, Tsukamoto S, Kaneko T, Imai H, et al. ING3 is essential for asymmetric cell division during mouse oocyte maturation. PLoS One 2013;8:e74749.

147. Huynh JR, St Johnston D. The origin of asymmetry: early polarisation of the Drosophila germline cyst and oocyte. Curr Biol 2004;14:R438-49.

148. Kim YM, Gang EJ, Kahn M. CBP/catenin antagonists: targeting LSCs' achilles heel. Exp Hematol 2017;52:1-11. 